\title{
A Graph-based Method for Timing Diagrams Representation and Verification
}

\author{
Viktor Cingel \\ Department of Computer Science and Engineering \\ Slovak Technical University, 82119 Bratislava, Slovakia
}

\begin{abstract}
A graph-based approach to the verification of timing constraints in timing diagrams is described. Timing diagrams as a specification tool together with a specialized theorem prover for inequalities are used to support the timing design process. The method for automatically proving the consistency of a designed timing diagram works over a graph representing the timing diagram and constraints. It is based on the detection of cycles in this graph. A simple extension of this method enables the generation of a set of timing constraints which have to be fulfilled in the given timing diagram.
\end{abstract}

\section{Introduction.}

In the design process of digital systems and circuits, a number of formal mathematical models for their description and the tools for automatic reasoning about their behaviour and implementation are being used currently. The functional verification seems to be the main task solved when designing a correct structural implementation from an external specification (usually expressed at the higher level). On the other hand, the timing and the way in which the system's interface has to operate in time are also very important design aspects [1,2]. Possible behaviours must satisfy both internal timing relations among building components and external timing requirements generated from the system's environment.

In general, the tools for the specification and verification of the system timing fall into four groups:

- tools supporting the timing analysis using critical paths detection $[4,9,17]$,

- tools performing timing simulation over a set of predefined patterns [14],

- tools generating timing constraints directly from the structure of a circuit $[5,6]$,

- tools producing mathematical proofs of certain timing properties $[1,7,8]$.

The method proposed in this paper falls into the fourth group of the tools. Recently, different formal approaches are studied to be employed in the timing verification. For example, formalisms based on HOL or the Boyer-Moore logic [7,13], timed event structures [15], or timed CSP-based notations [11].

When designing and verifying timing, we usually abstract from the functional specification. As a result we obtain the worst case timing behaviour satisfying all the necessary timing constraints. Timing diagrams usually provided by manufacturers describe timing aspects of the behaviour of components (chips). To specify precisely the external system timing, ignoring functional relationships and internal details, we 
first informally introduce a specification tool capable of describing timing diagrams. We use the min-max model for timing parameters which has proved to be sufficient for many practical applications. The formal reasoning on timing, which we apply in connection with a particular timing diagram, consists of two parts: proving theorems about the worst case behaviour in time, and the generation of timing constraints which must hold if the timing diagram is to be correct.

\section{Timing diagram as a specification tool}

\subsection{Basic definitions}

Time interval. The time interval $T$ is defined as a finite interval of real numbers representing continuous time. Any event occurrence time falls inside $T$.

System variables. The digital circuit the timing of which is to be analysed is externally specified by a set of system variables $S V=\left\{x_{p}, x_{2} \ldots, x_{n}\right\}$, each of them is of certain data type, denoted by $D x$. Each $D x$ also contains an unknown (or unspecified) value $\boldsymbol{u}$ of a given data type. To denote the behaviour in time, each variable $x$ is defined as a time function $x: T \rightarrow D x$.

Events. An event is defined as an instantaneous value change which occurs in time at certain variable. For example, the rising and successive falling edge represent two successive events. Events can be of various types. The type of an event $e$ for a variable $x$ we define by $\left.x<v_{s} v_{f}\right\rangle, v_{s} \neq v_{f}$, which denotes a value change from $v_{s}$ to $v_{f}$ of the $x$ 's data type. The most frequent event types are the following ones:

$x<0,1>$ up. $x$ - the rising edge of a boolean variable $x$,

$x<1,0>d w x$ - the falling edge of a boolean variable $x$,

$x<u, V\rangle u V x$ - the value change up to a valid numerical or symbolic value $V$, $x<V, u>d V x$ - the value change down from a valid value $V$.

In the sequel we use the abbreviated notation, e.g. up. $x$ instead of $x<0,1>$. By $E V$ we denote the set of all events existing in a particular timing diagram we analyse.

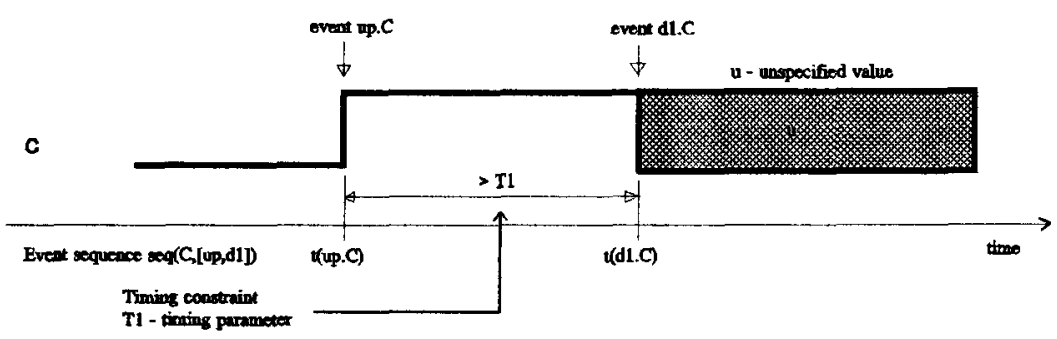

Fig.1. Basic items of a timing diagram 
Sequence of events. Individual events can form sequences to denote successive value changes occurring at a given variable in time. We will use sequences of events to describe pieces of a timing diagram (Fig.1). If necessary, in a particular sequence we can mark identical event type occurrences by different indexes in order to distinguish them as different events. A particular finite sequence of events occurring at a variable $x$ is specified by the predicate $\operatorname{seq}\left(x,\left[e_{1}, e_{3}, \ldots, e_{k}\right]\right)$ :

$$
t\left(e_{i}\right)<t\left(e_{i+1}\right), \text { for } i=1,2, \ldots, k-1
$$

The event occurrence time function specifies the time point at which a particular event has occurred: $t: E V \rightarrow T$ (Fig.1).

Timing constraints. Timing constraints specify quantitative timing relations among particular event occurrence times. Sometimes they are referred to as delays or restrictions. Both are usually drawn as arrows in a manufacturer's diagram coupled with a table specifying the possible ranges of values of timing parameters used (Fig.2). The simplest timing constraint can be specified by a relation

$$
t(e)+p \operatorname{rel} t(f)
$$

where

$e, f$ are events,

$p \quad$ is a timing parameter (see below), and

rel is one of the relations $=, \leq,<$.

However, more complex timing constrains can take place in a timing diagram. We will deal with them in the next section.

Timing parameters. In general, a timing parameter can be formalised by a function of a variety of physical parameters (e.g. time, temperature, fan-out, and so on). We will consider the following kinds of timing parameters only:

- a fixed, known numerical value $p$ (e.g. $p=5.3 \mathrm{~ns}$ ),

- a min-max model of a range $<p_{\text {mirp }} p_{\max }>$ (e.g. $p$ is from $<3.5,4.6>\mathrm{ns}$ ),

- a symbolic (unspecified) value $p$.

\subsection{Timing specification using timing diagrams}

\subsubsection{Formal specification of timing diagrams}

A timing diagram usually consists of:

- a set of event sequences defined by $\operatorname{seq}(x[\ldots])$ for particular variables,

- timing constraints defined among event occurrence times in that sequences. These are defined by a formula built of timing constraints predicates (see below).

From the above statements we can specify two kinds of timing constraints: 
- timing constraints specifying the relative ordering of events in time (see (1)),

- quantitative timing constraints which specify the necessary timing requirements imposed on event occurrence times. These constraints are prescribed by the manufacturer to ensure a correct functionality of a chip or component (e.g. the set-up time constraint)

Formally, timing constraints we specify by means of the predicates (for simplicity, called timing rules). Let $e, f, e_{1}, e_{2} \ldots$ be events, the symbol $\equiv$ stands for "is defined", and $p, p_{1}, p_{2} \ldots$ be timing parameters.

Equality rules (usually referred to as delays):

$$
\begin{array}{ll}
\operatorname{del}(e, f, p) \equiv t(e)+p=t(f) & \text { the single delay } \\
\operatorname{del}\left(m,\left[e_{1}, e_{2} \ldots, e_{r}\right], e,\left[p_{b}, p_{2} \ldots, p_{r}\right]\right) \equiv & \text { the multiple delay } \\
t(e)=m\left(t\left(e_{1}\right)+p_{b}, \ldots, t\left(e_{r}\right)+p_{r}\right), &
\end{array}
$$

where $\left[e_{1}, e_{2} \ldots, e_{r}\right]$ is the list of input events, $e$ is the output event, $\left[p_{l}, p_{3} \ldots, p_{r}\right]$ are particular timing parameters associated with input events, and the function $m$ is from $\{\min , \max \}, m: T \rightarrow T$ is used to choose either the earliest or latest occurrence time of the output event $e$ in compliance with the times of $e_{i}$ and parameters $p_{i}$. The multiple delay rule is often used to precisely model propagation delays in combinational circuits with different input/output delays.

Inequality rules (usually referred to as restrictions). They bind two event occurrence times with the timing parameter $p$ by the inequality relation.

$$
\begin{aligned}
& \text { aft }(e, f, p) \equiv t(e)+p \leq t(f) \\
& \operatorname{aftn}(e, f, p) \equiv t(e)+p<t(f)
\end{aligned}
$$

The stability rule specifies that a variable $x$ is assigned a stable value $V$ on the whole interval. It can equally be specified by the sequence $s e q(x,[u V, d V])$ and a proper set of timing rules which define that these two events can occur on the boundaries of the interval $T$ only (i.e. all other events must occur inside this interval).

$$
\operatorname{stb}(x, V) \equiv \forall t \in T: x(t)=V
$$

Example 1. The circuit consists of a simple logic block LOG and of a level-sensitive latch $\mathbf{L}$ (Fig.2). The task is to analyse several timing constraints imposed on the input port $A$ and the output port $C$ in compliance with the timing attributes of the clock signal $C L$ and internal timing restrictions (see the next section). The behaviour in time of this circuit is depicted in the timing diagram (Fig.2). In the diagram in Fig.2 one can see how timing constraints and parameters are related to the event sequences. The formal specification of this timing diagram is the following: 
SEQUENCES

$\operatorname{seq}(A,[u v, d V])$.

$\operatorname{seq}(B,[u v, d v])$.

$\operatorname{seq}\left(C L,\left[u p, d w, u p^{1}\right]\right)$.

$\operatorname{seq}(c,[u v, d v])$.
TIMING RULES

del( UV.A, UV.B, DLM ) •

del (dV.A, dV.B, DLm).

aft ( uV.B, dw.CL, Tset) .

aft (dw.CL, dV.B, Thld) .

aft ( up.CL, dw.CL, TI) .

aft (dw.CL, up ${ }^{1} . C L$, TO) .

del ( $\max ,[u p . C L, u V . B], u v . C,[D C M, D D M])$.

$\operatorname{del}\left(\mathrm{up}^{1} . \mathrm{CL}, \mathrm{dV} . \mathrm{C}, \mathrm{DCm}\right.$ )

where

- $u p^{l}$ stands for the second $u p . C L$ event in the sequence $\operatorname{seq}\left(C L,\left[u p, d w, u p^{l}\right]\right)$

- the timing parameters have the following meaning [16]:

$D L M, D L m$ - the maximum and minimum delays of LOG from $A$ to $B$,

Tset, Thld - the set-up and hold times,

$T 0, T 1 \quad$ - the minimum width of low and high levels of the clock signal $C L$,

$D C m, D C M$ - the minimum and maximum delays on the output of the latch,

$D D M \quad$ - the maximum delay from the input $B$ to the output $C$ of the latch.

$\mathbf{A}$
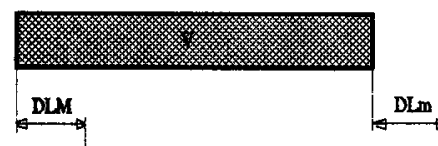

B
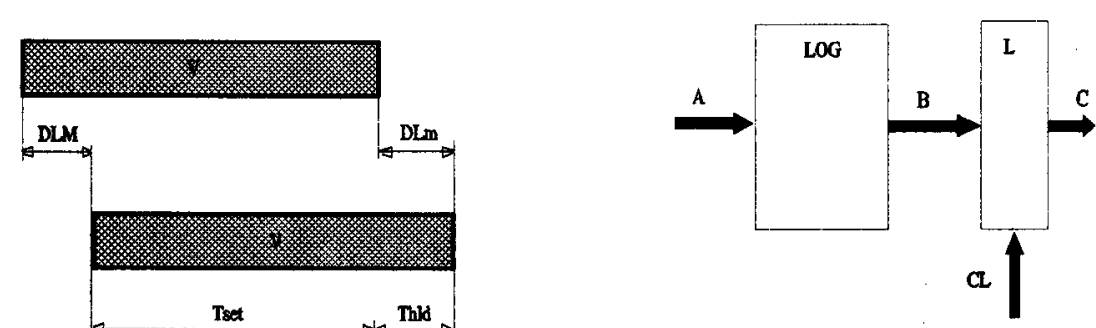

a

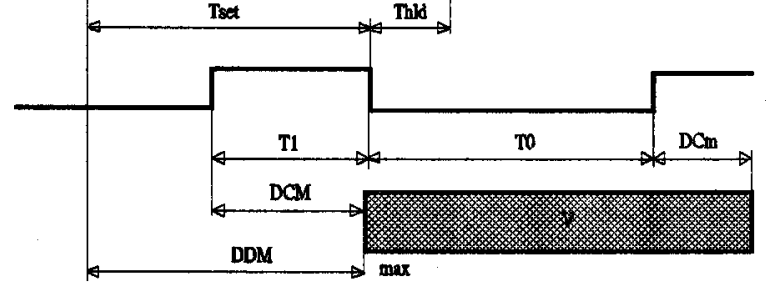

Fig.2. A simple sequential circuit and its timing

\subsubsection{Graphical representation of timing diagrams}

The proving method is based on an algorithm for finding out cycles in the graph [8], which represents the timing diagram and the appropriate correctness theorem. In the first step, therefore, we have to construct a finite directed graph $G=\langle N, E\rangle$. Nodes in $N$ represent events, and edges (or arrows) in $E$ represent timing constraints. 
Informally, nodes are generated from the event occurrences involved in the predicates $\operatorname{seq}\left(x_{i}\left[e_{b}, e_{2} \ldots, e_{k}\right]\right)$. If a node is assigned to an event $e$ which appears in some predicate $\operatorname{del}\left(m,\left[e_{b}, e_{2} \ldots, e_{r}\right], e,\left[p_{b}, p_{2} \ldots, p_{r}\right]\right)$ then to this node also the functional symbol $m$ is assigned. For the most frequent situations the node transformation process is depicted in Fig.3, Fig.4, and also in Fig.5. for the timing diagram specifying the timing behaviour of the simple circuit from Fig.2.

In the second step, we have to include edges into the graph. Look at the Fig.4. in order to understand the rules for generating edges from the predicates. The edges can be of two types:

- single (or unidirectional) arrows which are derived from timing constraints given in the form of inequalities (from aft(.) and aftn(.) predicates, and from seq(.) predicates). These arrows can be traversed only in one direction.

- double (or bidirectional) arrows which are derived from timing constraints given in the form of equalities (from del(.) predicates). To each bidirectional edge an orientation label ' + ' is assigned (see Fig.4) in order to distinguish the position of the events in a particular equality. Bidirectional edges can be traversed in both directions. The orientation given by ' + ' is important when the proving procedure generates cycles (see Section 3.3.2).
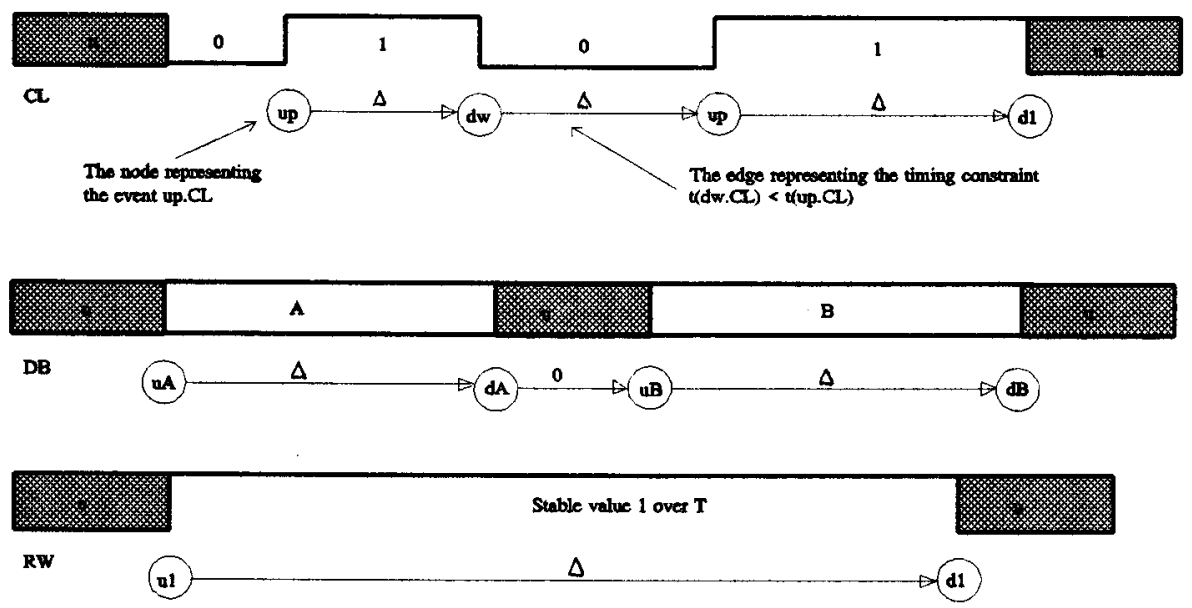

Fig.3. Transformation of event sequences into a graphical representation

It is important to stress that a single arrow labelled with the timing parameter $p$, and connecting two nodes labelled with events $e$ and $f$, represents the inequality [8]:

$$
t(e)+p \leq t(f)
$$

In order to represent the inequality in the form

$$
t(e)+p<t(f)
$$


by the single (unidirectional) arrow, we add a non-zero timing parameter $\Delta>0$ (tending to zero) to $p$. Therefore the last inequality is finally represented by an arrow labelled by the timing parameter $p+\Delta$ (see Fig.4).

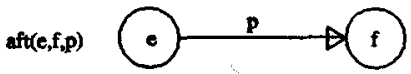

$t(e)+p \leqslant t(f)$

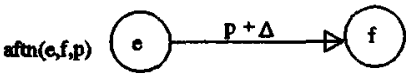

$t(e)+p+\Delta \leqslant t(t)$ $\operatorname{det}(\mathbf{e}, \mathbf{f}, \mathbf{p})$

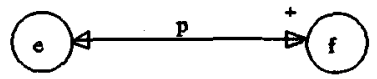

$t(e)+p=t(f)$

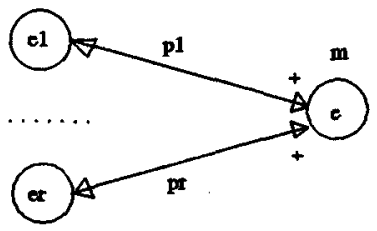

$t(0)=m(t(01)+p 1, \ldots, t(e x)+p t)$

Fig.4. Transformation of timing constraints into a graphical representation

In the verification process, the final graph used by the reasoning method includes nodes and edges extracted from different timing specifications (timing diagrams) of components as well as from other assumptions stated for a particular design problem.

\section{Formal reasoning on timing in a timing diagram}

\subsection{General methodology}

When the timing diagram for a particular design problem is built up, the designer usually wants to prove its certain properties and/or to optimise the performance of the system in time. The sequence of main design activities can be formalised and solved in the following way:

- Let $T D_{\text {circuit }}$ denotes the formula representing the timing diagram for a particular timing behaviour of a circuit. $T D_{\text {circuit }}$ is transformed into a graph $G$.

- Let the formula $T C_{\text {input }}$ denotes timing constraints which influent the primary (interface) circuit inputs. The task is to prove the theorem

$$
T D_{\text {circuit }} \Rightarrow T C_{\text {input }}
$$

or to generate the conditions among timing parametrs that must be met.

- When the input timing constraints $T C_{\text {input }}$ are known and understood, we should prove certain timing constraints $T C_{i o}$ emerging between primary inputs and outputs. At this moment we know that the circuit behaves according to the timing 
specified by $T D_{\text {circuit. }}$ If necessary, from $T C_{\text {input }}$ already known, the designer can prescribe certain assumptions on the input timing. Let $T C_{\text {wserin }}$ specifies such assumptions. Hence, the final theorem is

$$
T D_{\text {circuit }} . T C_{\text {userin }} \Rightarrow T C_{i o}
$$

Note that all mentioned subformulae are formed from predicates defining certain timing constraint among events on interface variables. Except of the cycle detection, the proving procedure [8] uses the David-Putnam method to prove unsatisfiability of the negation of the original theorem. For (10) and (11), the formulae

$$
\begin{aligned}
& T D_{\text {circuit }} \cdot \neg T C_{\text {input }} \\
& T D_{\text {circuit }} . T C_{\text {userin }} . \neg T C_{i o}
\end{aligned}
$$

have to be proved to be unsatisfiable with respect to the intervals of timing parameters used in them. The original theorems are then valid.

\subsection{A proving procedure - summary}

It should be obvious that the above presented theorems $(10,11)$ consist of: (i) inequality relations $t(e)+p \leq t(f)$ (from $\operatorname{seq}(),. a f t(),. a f t n($.$) and s t b($.$) predicates),$ (ii) equality relations $t(e)+p=t(f)$ (from $\operatorname{del}($.) predicates), and (iii) logical connectives like . (and), + (or), $\neg$ (not). If we construct a graphical representation of the theorem to be proved, and we rewrite the theorem into its propositional variant, then it is possible to apply a very efficient procedure [8] to check unsatisfiability of the negation of the original theorem. We give a very brief summary of this procedure followed by the description of our extended variant.

Let $F$ be a formula unsatisfiability of which we want to determine (e.g. one of (12)). Clearly, $F$ consists of inequality and equality relations (e.g. $t(e)+p \leq t(f))$ and logical connectives. This atomic inequality is called a literal $L$. Each literal in $F$ can be graphically represented by a directed edge labelled by $p$ (see Fig.4) and connecting two nodes labelled by $e$ and $f$, respectively. Let $G$ be such a graph representing $F$. Let $F_{p}$ be a propositional variant of $F$ obtained in such a way, that each literal $L_{i}$ in $F$ is represented by a single propositional variable $A_{i}$ in $F_{p}$. Logical connectives are preserved. The proving procedure then works in two steps:

- Search for cycles in $G$ to find cycles with positive weights, assuming that edge parameters are integer constants. The weight is defined as the sum of parameters along the edges involved in the cycle. The conjunction $\left(A_{i}, A_{2} \ldots . A_{n}\right)$ of literals (i.e. originally inequalities) involved in such a cycle is unsatisfiable.

- For each positive cycle in $G$ construct a disjunctive clause $C=\left(\neg A_{1}+\neg A_{2}+\right.$ $\ldots+\neg A_{n}$ ), which is true. This clause contains negations of propositional variables which belong to literals involved in the cycle with the positive weight. Then, this always true clause is used to refute $F_{p}$ (i.e. the propositional variant of $F$ ). Consequently, $F_{p}$ is satisfiable if and only if $F_{p} . C$ is satisfiable. We have used the Davis-Putnam method [10] to check satisfiability. 


\subsection{An extended proving procedure}

In our formalism we have introduced timing relations in the form of equalities, namely, the single del(.) predicate and the multiple delay represented by $\operatorname{del}(m, .$. predicate. Another reason for extending this procedure is the fact that we have defined timing parameters by the min-max model. Thus the above discussed procedure requires certain extensions.

\subsubsection{Propositional variant of the original theorem}

After having transformed the formula to be proved into its graphical representation (see Section 2.2.2), the next step is to create a propositional variant $F_{p}$ of $F . F_{p}$ is used in the refutation process. In our model the following steps have to be performed:

- each predicate $\operatorname{aft}(),. \operatorname{afth}($.$) , and the single \operatorname{del}($.$) is uniquely represented by a$ fresh propositional variable $A$ in $F_{p}$,

- each rule $\operatorname{del}\left(m,\left[e_{b}, e_{3} \ldots, e_{r}\right], e,\left[p_{b}, p_{3} \ldots, p_{r}\right]\right)$ is represented by a clause $\left(A_{1}+A_{2}\right.$ $+\ldots+A_{n}$ ) where each propositional variable $A_{i}$ represents one single equality relation $t(e)=t\left(e_{i}\right)+p_{i}$ (see (4)). The function $m$ is assigned to the output node,

- logical connectives are preserved.

\subsubsection{The cycle detection problems}

The main problem is to detect cycles with positive weights in $G$. In our model, a set $C$ of edges forming a cycle in the graph $G$ consists of two disjunctive subsets $C+$ and $C$ - of edges traversed in the positive or negative orientation respectively. Intuitively:

- all unidirectional edges and the bidirectional edges traversed towards the end nodes marked by "+", fall into $C+$,

- all the bidirectional edges traversed from the nodes marked by "+ "fall into $C$ The minimum weight $w_{\min }$ of a cycle $C$ is then defined as the sum of min-max values:

$$
w_{\min }(C)=\sum_{C+} p_{\min }-\sum_{C-} p_{\text {max }}
$$

The nodes and corresponding edges generated from the multiple del $(m, .$.$) predicates$ have an important property which strongly eliminates the cycles with positive weights we have to search for. Let $\operatorname{del}\left(m,\left[e_{l}, e_{2}, \ldots, e_{r}\right], e,\left[p_{1}, p_{2} \ldots, p_{r}\right]\right)$ be a multiple delay appearing in $F$. After generating a corresponding piece of $G$ (see Fig.4) we obtain the following set of edges $\left\langle e_{p}, e\right\rangle,\left\langle e_{y} e\right\rangle, \ldots,\left\langle e_{n} e\right\rangle$ and corresponding nodes labelled by events. The node labelled by $e$ is assigned a function $m$.

Theorem. If a cycle $C$ in $G$ contains two edges generated from one multiple delay timing rule, then this cycle is not the positive one.

Proof. Follows from the semantics of the $\min$ or $\max$ function $m$ which always adjusts the weight $w_{\min }(C)$ to 0 . The conjunctive clause $C$ discussed in Section 3.2. is therefore always satisfiable. 
The conclusion is that into the algorithm for finding all cycles in $G$ we have to add a rule which prevents us from generating cycles with edges belonging to multiple delay timing rules. Having generated a set of cycles with $w_{\min }(C)>0$, we have to refute the original formula using the approach described in Section 3.2.

\subsubsection{An algorithm - a general shape}

Considering the fact that the time required for the verification depends mainly on the cycle detection and on the size of the graph, we reduce the graph size and then we generate all possible cycles merely once. This is then followed by a possible repetitive proof of the theorem with different intervals of timing parameters values. The draft of the algorithm is given below.

\section{Algorithm}

1. From the timing diagram, construct the formula $F$ (and its negation) to be verified; set appropriate intervals of initial (or intended) values of timing parameters;

2. Construct a graph $G$ and a propositional formula $F_{p}$ from $F$; use reduction rules to reduce the size of $G$ and the length of $F$ as much as possible (e.g. to eliminate all non-essential nodes);

3. Generate the set $S C$ of all cycles in the graph $G$;

\section{REPEAT}

4. evaluate weights of all cycles in SC with given min-max values of timing parameters;

5. use all the cycles with the positive weights to refute the formula $F_{p}$

6. choose new values of timing parameters if necessary;

UNTIL satisfactory values of timing parameters are obtained.

Once the theorem is proved, we do not need to verify the given timing description for all possible combinations of values of the timing parameters. The result of such a proof is independent of occurrence times of particular events.

In most cases a timing specification is given merely in the form of conjunction of timing rules. For such cases we introduce a simple step into the algorithm which generates timing constraints among extreme values of timing parameters. This new step replaces the REPEAT-UNTIL part in this algorithm. The generated timing constraints must hold in order to ensure validity of the original timing description. For each cycle found in the graphical representation of such a conjunction, the following constraints must be satisfied:

$$
w_{\max }(C)=\sum_{C_{+}} p_{\max }-\sum_{C-} p_{\min } \leq 0 \cdots \sum_{C_{+}} p_{\max } \leq \sum_{C-} \sum_{p_{\min }}
$$

After generating a set of possible constraints, it is useful to minimize this set. From the semantics of inequalities we have proposed several reduction rules to eliminate redundant and always satisfied constraints. In general, the rules are divided into two 
main groups: (i) Rules which eliminate timing parameters and subexpressions in the set of timing constraints on the algebraic level (i.e., symbolically), (ii) rules which utilize information on specific (known) values of timing parameters.

For the cycle detection, we have used and modified an efficient procedure described in [12]. Before the cycle detection procedure is started, all non-essential nodes and corresponding edges are removed from the graph (e.g., nodes with only incoming or outgoing edges). We have implemented the presented verification procedures in the Pascal language. These procedures were integrated into an experimental version of a timing diagram editor. Proofs are executed within the editor's enviroment with the possibility to show violated constraints.

\section{Verification examples}

\subsection{A simple sequential system}

The best way how to understand the proposed approach is to highlight the particular verification steps on an example. We continue with the example of the simple sequential circuit illustrated in Fig.2. Suppose, that we want to know the timing constraints imposed on the duration of a valid value $V$ on the interface variable $A$ with respect to the clocking signal $C L$. This valid value $V$ must be valid some time before the falling edge at $C L$ occurs, and must remain stable some time after this edge. The formula specifying these requirements can be formed as the conjunction

$$
\begin{aligned}
T C_{\text {input }} \equiv \quad & \text { aft }(u V \cdot A, d w \cdot C L, T S A) . \\
& \text { aft }(d w \cdot C L, d V \cdot A, T H A)
\end{aligned}
$$

where the timing parameters TSA and THA specify the neccessary amount of time measured between particular event occurrence times. Let $T D_{\text {circuit }}$ be the formula representing the timing diagram (see (7)). We can decompose the verification problem into two steps using the logical rule $(A \Rightarrow(B . C))=(A \Rightarrow B) .(A \Rightarrow C)$. It is easy to show that the negation of the predicate aft $(e, f, p)$ is aftn $(f, e,-p)$. The negation of the final theorem for one case is

$$
\left.T D_{\text {circuit }} \text { aftn( } d w . C L, u V . A,-T S A\right)
$$

which must be unsatisfiable. The same is done for the second subformulae. First, we have to build up a graphical representation of this formulae. The corresponding graph is shown in Fig.5. where the dashed lines represent the negations of the previously commented input timing constraints.

The procedure for detecting cycles in this graph finds three cycles. They are also depicted in the Fig.5. From these three cycles the following timing constraints are generated and/or can be evaluated for chosen values of timing parameters: 
Cycle 1: $\quad-T S A+T s e t+D L M \leq 0$

Cycle 2: $\quad-T H A+$ Thld $<D L m$

Cycle 3: $\quad-T H A-T S A<0$

If for chosen min-max values of timing parameters the weight $w_{\min }(C)$ of some cycle is evaluated as positive, then the procedure has to prove unsatisfiability of the negation of the original theorem taking into account such positive cycles. It is important to note, that after proving the consistency of the original timing diagram, the verification results are independent of concrete values of event occurrence times. The consistency of relations among timing parameters ensures the correct behaviour of the circuit under the conditions specified by the theorem.

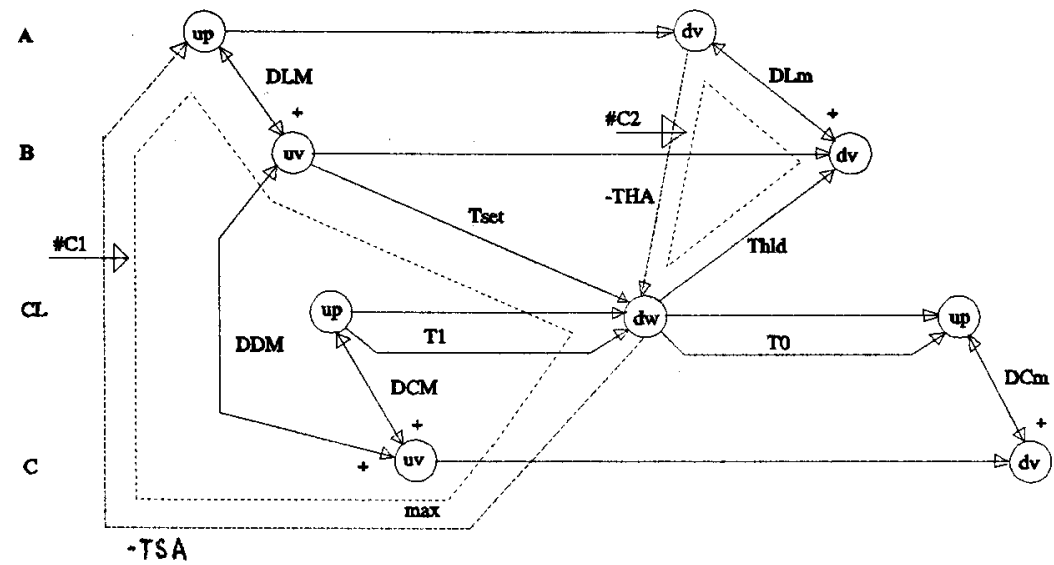

Fig.5. Input timing constraints verification: two possible cycles

\subsection{Verification results}

We have tested our PC-based implementation on several examples of well-known circuits. The table below summarises the results of the verification. The first circuit is the sequential circuit discussed in this paper. The second circuit is a more complicated bus arbiter which controls access to a bus from two sources. In the last example we have verified the timing in the WRITE operation of an 8080 system which consists of the standard set of components $(8080,8205,8224,8228)$, and additional components (seven gates, two D-flip-flops, and one semicustom chip) [3].

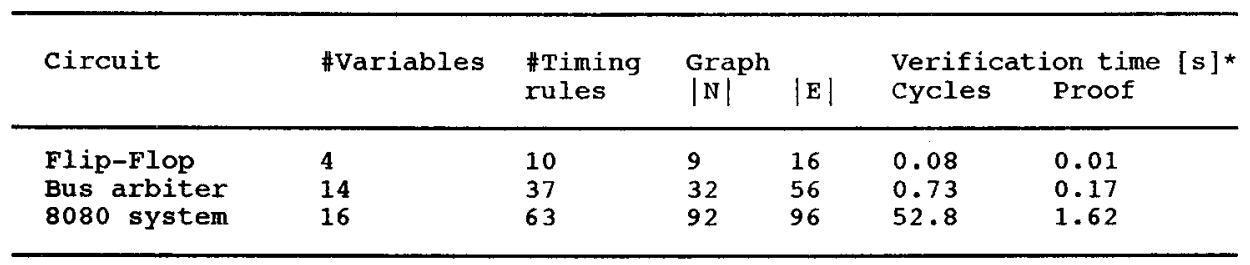

(* measured on an IBM $38633 \mathrm{MHz}$ machine) 


\section{Conclusion and future work}

We have presented a method for the formal specification of the timing diagrams which is used to describe the external behaviour of circuits and systems from the timing point of view. The specification method allows the designer to organize his/her design in a hierarchical fashion by ignoring internal timing and functional details. As the verification method based on the detection of cycles is fully automated and it has shown reasonable efficiency, it seems to be an acceptable candidate for the incorporation into the conventional CAD tools designated to timing design. We suggest several ways in which the method could be extended and improved: to organize libraries of timing specifications provided by manufacturers which can easily be accessed by the timing diagram editor; to modify the proving procedure to allow reasoning on timing with timing parameters modelled by symbolic names; to use other general purpose theorem provers (e.g., the HOL system) to reason on timing constraints generated from the graph, and so on. Some of the proposed topics are intended to be the aim of the future research.

\section{References}

1. Antoine,C.-Goff,B.L.: Timing Diagrams for Writing and Checking Logical and Behavioural Properties of Integrated Circuits. In. Proc.of Advanced Research Workshop on Correct Hardware Design Methodologies, Turin, June 1991.

2. Borriello,G.-Katz,R.H.: Synthesizing Transducers from Interface Specifications. In. Proc. of VLSI'87 (C.H.Sequin edt.), Elsevier Sc. Publisher, 1988, pp.403-418.

3. Cingel,V.: Specification and Verification of Timing in Digital Systems. Ph.D. Thesis, Department of Computer Science and Engineering, Slovak Technical University, Bratislava, Jan 1991, 175 pp (in Slovak).

4. Claesen,L. et al.: Efficient False Path Elimination Algorithm for Timing Verification by Event Graph Preprocessing. Integration, the VLSI journal, Vol.2, 1989, pp.173-188.

5. Dagenais,M.R.-Rumin,N.C.: Automatic Determination of Optimal Clocking Parameters in Synchronous MOS VLSI Circuits. In. Advanced in CAD for VLSI, Vol.5, 1987, pp.20-33.

6. Eveking,H.-Mai,Ch.: Formal Verification of Timing Conditions. In. Proc. of 1st EDAC, Glasgow, 1990, pp.512-517.

7. Herbert,J.: Formal Verification of Basic Memory Devices. In. Formal VLSI Correctness Verification, (L.Claesen edt.), Elsevier Sc. Publisher, 1990.

8. Jahanian,F.-Mok,A.K.L.: A Graph-Theoretic Approach for Timing Analysis and its Implementation. IEEE Tr. on Computers, C-36, No.8, Aug 1987, pp.961-975.

9. Jouppi, N.P.: Timing analysis and Performance Improvement of MOS VLSI Designs. IEEE Tr. on CAD, No.4, 1987, pp.650-665.

10. Loveland,D.W.: Automated theorem proving: A logical basis. Fundamental studies in Computer Science Vol.6., North-Holland, 1978. 
11. Reed,G.M.-Roscoe,A.W.: A Timed Model of Communicating Sequential Processes. In. ICALP-86, LNCS-226, Springer Verlag, 1986.

12. Reingold,M.E.: Combinatorial Algorithms. Theory and Practice. Prentice Hall, 1977, $441 \mathrm{pp}$.

13. Salem,A.: Formal Reasoning on Timing Constructs in VHDL. (Lecture Notes), presented at Advanced Research Workshop on Correct Hardware Design Methodologies, Turin, June 1991.

14. Seger,C.J.-Bryant,R.E.: Modeling of Circuit Delays in Symbolic Simulation. In. Formal VLSI Correctness Verification (L.Claesen edt.), Elsevier Sc. Publisher, 1990, pp. 23-37.

15. Subrahmanyam,P.A.: What's in Timing Discipline ? Considerations in the Specification and Synthesis of Systems with Interacting Asynchronous and Synchronous Components. In. Hardware Specification, Verification and Synthesis: Mathematical Aspects. (eds. M.Leeser, G.Brown), LNCS-408, Springer Verlag, 1990, pp.202-223.

16. Unger,S.H.-Tan,C.J.: Clocking Schemes for High-Speed Digital Systems. IEEE Tr. on Computers, C-35, No.10, Oct 1986, pp.880-895.

17. Wallace,D.E.-Sequin,C.H.: ATV: An Abstract Timing Verifier.In. Proc. of 26th ACM/IEEE Design Automation Conference, 1989, pp.655-661. 